\title{
Three-Year Outcomes in Deep Brain Stimulation for Highly Resistant Obsessive-Compulsive Disorder
}

\author{
Benjamin D Greenberg*, 1,2, Donald A Malone ${ }^{3,4}$, Gerhard M Friehs ${ }^{1,2}$, Ali R Rezai, ${ }^{3,4}$, Cynthia S Kubu ${ }^{3,4}$, \\ Paul F Malloy ${ }^{1,2}$, Stephen P Salloway ${ }^{1,2}$, Michael S Okun ${ }^{5,6}$, Wayne K Goodman ${ }^{5,6}$ and Steven A Rasmussen ${ }^{1,2}$ \\ 'Departments of Psychiatry and Human Behavior, Brown Medical School, Butler Hospital and Rhode Island Hospital, Providence, RI, USA; \\ ${ }^{2}$ Department of Clinical Neurosciences (Neurosurgery Division), Brown Medical School, Butler Hospital and Rhode Island Hospital, Providence, \\ RI, USA; ${ }^{3}$ Department of Psychiatry, Cleveland Clinic Foundation, Cleveland, OH, USA; ${ }^{4}$ Center for Neurological Restoration, Cleveland Clinic \\ Foundation, Cleveland, OH, USA; ${ }^{5}$ Departments of Psychiatry and Neurology, University of Florida, Gainesville, FL, USA; ${ }^{6}$ Movement Disorders \\ Center, University of Florida, Gainesville, FL, USA
}

Deep brain stimulation (DBS) of the anterior limb of the internal capsule has been shown to be beneficial in the short term for obsessive-compulsive disorder (OCD) patients who exhaust conventional therapies. Nuttin et al, who published the first DBS for OCD series, found promising results using a capsule target immediately rostral to the anterior commissure extending into adjacent ventral capsule/ventral striatum (VCNS). Published long-term outcome data are limited to four patients. In this collaborative study, 10 adult OCD patients meeting stringent criteria for severity and treatment resistance had quadripolar stimulating leads implanted bilaterally in the VCNS. DBS was activated openly 3 weeks later. Eight patients have been followed for at least 36 months. Group Yale-Brown Obsessive Compulsive Scale (YBOCS) scores decreased from 34.6 \pm 0.6 (mean \pm SEM) at baseline (severe) to $22.3 \pm 2.1$ (moderate) at 36 months $(p<0.00 \mathrm{I})$. Four of eight patients had a $\geqslant 35 \%$ decrease in YBOCS severity at 36 months; in two patients, scores declined between 25 and 35\%. Global Assessment of Functioning scores improved from 36.6 \pm 1.5 at baseline to $53.8 \pm 2.5$ at 36 months ( $p<0.00 \mathrm{l}$ ). Depression and anxiety also improved, as did self-care, independent living, and work, school, and social functioning. Surgical adverse effects included an asymptomatic hemorrhage, a single seizure, and a superficial infection. Psychiatric adverse effects included transient hypomanic symptoms, and worsened depression and OCD when DBS was interrupted by stimulator battery depletion. This open study found promising long-term effects of DBS in highly treatment-resistant OCD.

Neuropsychopharmacology (2006) 31, 2384-2393. doi: I 0.1038/sj.npp. I 30 I I 65; published online 19 July 2006

Keywords: deep brain stimulation; obsessive-compulsive disorder; internal capsule; long-term treatment; neurosurgery

\section{INTRODUCTION}

Severe, treatment-resistant obsessive-compulsive disorder (OCD) is a chronic, debilitating disorder, imposing considerable suffering and markedly impairing affected individuals' ability to work, interact socially, or live independently. Therapeutic options in this group were previously limited to ablative surgery, such as anterior capsulotomy (see review by Greenberg et al, 2003) or anterior cingulotomy (Dougherty et al, 2002). Beginning with the work of Nuttin et al, small-scale controlled (Nuttin et al, 1999, 2003b; Gabriels et al, 2003; Abelson et al, 2005) and open studies (Anderson and Ahmed, 2003; Aouizerate

*Correspondence: Dr BD Greenberg, Departments of Psychiatry and Human Behavior, Brown Medical School, Butler Hospital and Rhode Island Hospital, 345 Blackstone Blvd., Providence, RI 02906 USA, Tel: + | 40| 455 6602, Fax: + | 40| 4556442

E-mail: bgreenberg@butler.org

Received 24 January 2006; revised 2 May 2006; accepted II May 2006 Online publication: 9 June 2006 at http://www.acnp.org/citations/ Npp060906060045/default.pdf et al, 2004; Sturm et al, 2003; Aouizerate et al, 2005) have suggested that deep brain stimulation (DBS) of the internal capsule and/or the adjacent ventral striatal region may be of benefit to severely affected OCD patients who have exhausted conventional therapies. The implantation sites were all generally similar to those for anterior capsulotomy lesions, but the specific targets, surgical approaches, and electrode designs have varied. The specific target used by Nuttin et al, whose results have been particularly promising, was based in part on our own gamma knife capsulotomy work (Rasmussen, in preparation). The target for gamma capsulotomy is within the ventral half of the anterior limb of the internal capsule, impinging inferiorly on the ventral striatum. We refer to it as the ventral capsule/ventral striatum (VC/VS) site. Determining the long-term efficacy and safety outcomes of VC/VS, DBS are particularly important in determining its viability as a therapy. Longterm effects of VC/VS DBS have been reported in a single published study totaling three patients who had DBS for at least 21 months (Nuttin et al, 2003b). The purpose of this study was to examine the outcomes of DBS over 3 years in 
patients with severe, treatment-resistant OCD, in multiple domains of interest. These included severity of core OCD symptoms, affective and non-OCD anxiety pathology commonly comorbid with OCD, and global functioning.

\section{PATIENTS AND METHODS}

This was a collaborative study including the psychiatric neurosurgery groups at Butler Hospital (BH)/Brown Medical School and the Cleveland Clinic (CC). Each site enrolled five adult patients beginning in February 2001 after IRB and FDA Investigational Device Exemption approvals were obtained at each site. Patient selection was based on the criteria developed to determine eligibility for neurosurgery for otherwise intractable OCD (Dougherty et al, 2002) and followed the guidelines of the DBS for OCD Collaborative Group (Nuttin et al, 2003a).

Detailed patient screening, record review, interviews with treating clinicians, and baseline assessments were used to assure that OCD was the primary diagnosis (using the Structured Clinical Interview for DSM-IV) (First et al, 2001). The minimum level of OCD severity required for entry was a Yale-Brown Obsessive Compulsive Scale (YBOCS; Goodman et al, 1989) score of 28. Treatment resistance was defined as failure to obtain meaningful OCD improvement after pharmacotherapy, including adequate trials ( $\geqslant 3$ months, with doses at or, if tolerated, beyond the FDA maximum recommended dose) of at least three serotonin reuptake inhibitors (SRIs), one of which had to be clomipramine. Trials of augmentation of an SRI with a neuroleptic and with a benzodiazepine were required, as was a minimum of 20 sessions of therapist-guided behavior therapy (exposure and response prevention). In practice, all patients exceeded the number of treatments required to satisfy this entry criterion. In no case did sustained efforts at behavior therapy plus pharmacotherapy reduce symptoms to a tolerable level. All patients had chronic OCD, ranging from 11 to 39 years in duration. Patients were excluded if there was a history of a current or past psychotic disorder, a manic episode within the preceding 3 years, any current clinically significant neurological disorder or medical illness (except for tic disorders), any clinical significant abnormality on preoperative magnetic resonance imaging (MRI), any labeled DBS contraindication and/or inability to undergo presurgical MRI, current or unstably remitted substance abuse or dependence, pregnancy or lack of use of effective contraception in women of childbearing age, a clinical history of severe personality disorder, inability to adhere to the operational requirements of the study, and imminent suicidal risk. At each site, the evaluations and consent process were reviewed by an independent committee (including psychiatrists who were not connected with the study), which made final eligibility determinations. All patients were informed that lesion procedures, specifically gamma knife capsulotomy or anterior cingulotomy, were potential alternative treatments.

Patients (Table 1) were aged 21-58 (six men and four women). Presurgical baseline severity on the YBOCS was 32-38 (mean \pm SEM: $34.6 \pm 0.6$ ), and did not differ between the BH and CC groups. Eight of 10 patients had comorbid DSM-IV major depression. The BH patients scored significantly higher on the 24-item Hamilton Rating Scale for Depression (Hamilton 1967; HRSD-24; overall range: $16-29$; BH mean: 25.2; CC mean: 17.0 ; $\mathrm{F}(1,8)=32.96$, $p<0.001)$.

Of the 10 patients implanted, one died (owing to recurrent breast cancer) 9 months after implantation. Her data are not carried forward in the analysis. Eight patients have reached the 36-month rating point, whereas one has

Table I Patient Characteristics

\begin{tabular}{|c|c|c|c|c|c|c|}
\hline $\mathrm{BHI}$ & $32, M$ & 10 & 22 & 32 & CK, AR, CTM, PF & Y \\
\hline $\mathrm{BH} 2$ & $40, F$ & 16 & 24 & 34 & WSH, INC, HRD & Y \\
\hline $\mathrm{BH} 3$ & $39, M$ & 12 & 27 & 35 & CK, AR, GR, INC & Y \\
\hline $\mathrm{BH} 4$ & $26, F$ & 15 & 11 & 34 & INC, PF & Y \\
\hline $\mathrm{BH} 5$ & $32, M$ & 10 & 22 & 33 & CTM, DT, RE & Y \\
\hline $\mathrm{CC} 2$ & $35, F$ & 12 & 23 & 36 & INC, RP & Y \\
\hline $\mathrm{CC} 3$ & $22, M$ & 8 & 14 & 35 & SYM, INC, OFF, WSH & Y \\
\hline CC4 & $23, M$ & 7 & 16 & 33 & CK, SYM & Y \\
\hline CC5 & $45, M$ & 19 & 26 & 36 & INC, CTM & $N$ \\
\hline
\end{tabular}

$\mathrm{BH}=$ Butler Hospital; $\mathrm{CC}=$ Cleveland Clinic.

OCD symptom abbreviations: $C K=$ checking; $A R=$ arranging/ordering; $C T M=$ contamination fears; PF = perfectionism; WSH = washing; $M D D=$ comorbid DSM-IV depression; INC = 'incompleteness' (Rasmussen and Eisen, 1992); HRD = hoarding; GR = grooming rituals; DT=doubt; RE = reassurance seeking; FH= fear of harming others; RP = repeating; SYM = symmetry obsessions/compulsions; OFF $=$ fear of offending others.

apatient $\mathrm{CCl}$ died of recurrent breast cancer at 9 months. Her data were not carried forward in the analysis. 
just reached 24 months. Stimulation was discontinued in two patients, after 12 and 24 months, respectively, owing to lack of adequate therapeutic effects. Both continued to be followed and rated, with DBS off. As discontinuation of DBS in a proportion of patients is likely after longterm treatment, the data for these two patients, including the later time points with DBS off, were included in the analysis.

\section{Device Implantation}

The surgical target was the anterior limb of the internal capsule just anterior to the rostral border of the anterior commissure in the coronal plane. Figure 1 is a coronal image showing the postimplantation lead location in a representative patient. At least one investigator from the $\mathrm{BH}$ team (BDG or SAR) attended all the CC implantations to help assure comparability of targeting.

Two custom-made quadripolar stimulating leads were implanted stereotactically under MRI guidance, one on each side. The leads (Model 3387 IES, Medtronic Inc., Minneapolis, MN) were $1.27 \mathrm{~mm}$ in diameter. Each had four independently programmable cylindrical electrode contacts $3 \mathrm{~mm}$ in length, spaced $4 \mathrm{~mm}$ apart, numbered as contact 0 (most distal) to contact 3 (most proximal). The leads were implanted to follow the trajectory of the anterior capsule in the coronal plane. The dorsal-most contact (3) was at the dorsal margin of the anterior capsule. The distal contact (0) extended into the ventral striatum, in the caudal nucleus accumbens. Postoperative imaging (CT for BH patients; MRI for CC patients) was coregistered with the preoperative stereotactic MRI to localize the leads. See Figure 1 (legend) for postimplantation lead locations. No significant differences between implantation positions across the $\mathrm{BH}$ and $\mathrm{CC}$ study sites were apparent. On the same day, battery-operated, programmable implantable neurostimulators (INSs; Soletra model, Medtronic Inc.) were placed in the chest. One INS was connected to each brain stimulating lead by extension wires tunneled subcutaneously under general anesthesia.

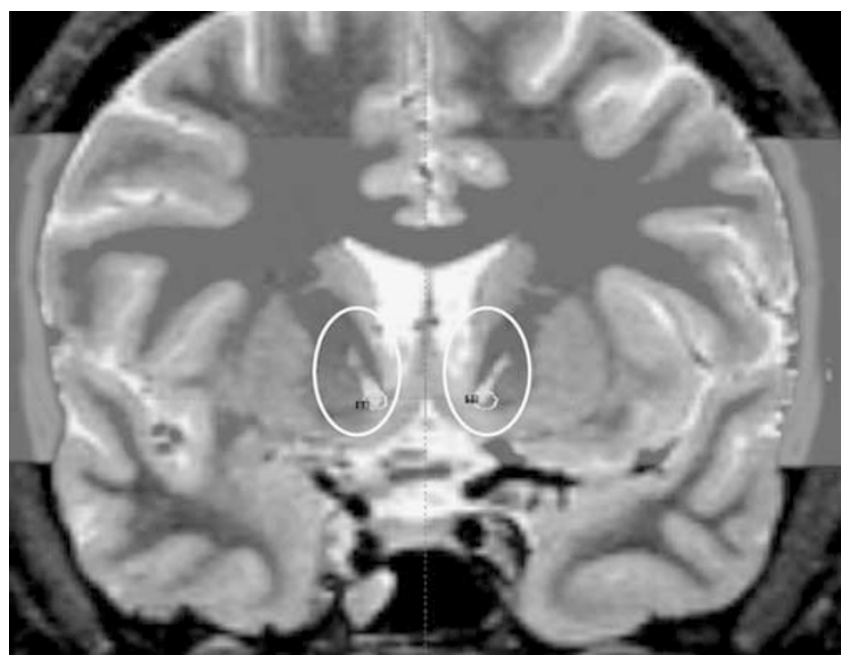

Figure I DBS lead location.

\section{Intraoperative Test Stimulation}

In the operating room, test stimulation was in the bipolar mode, starting with the ventral electrodes set active and negative against contact 3 (at the dorsal capsule margin) set positive. Stimulation was $130 \mathrm{~Hz}$, at pulse widths of 90 and $210 \mu \mathrm{s}$ and at $2-6 \mathrm{~V}$. The effects of intraoperative test stimulation and that of the subsequent monopolar survey of electrode contacts (technique described below) performed 2 weeks after implantation were generally similar. The most common effects were improvement in mood and anxiety, spontaneity, verbal fluency, and facial expressiveness, together with increased alertness and heart rate (a maximum increase of $12.6 \pm 2.7$ (range 6-20) beats per minute across patients). Patients, who were unaware of intraoperative test conditions, were asked to report their mood, anxiety, and alertness verbally using 10-point scales. Qualitatively similar effects were elicited by the activation of electrode contact 0 (in the ventral striatum), electrode 1 (within the ventral capsule white matter), and electrode 2 (near the dorsal-ventral midpoint of the capsule in the coronal plane). However, anxiety and flushing were more common with stimulation of the most ventral contact (0). Setting the most dorsal contact (3) active and negative generally produced a distinctly different pattern of less change in any domain, and sometimes, in contrast to ventral cathodal stimulation, a sense of decreased alertness or cognitive dulling. All the above acute effects of DBS reversed when stimulation stopped.

\section{Monopolar Survey}

An average of 3 weeks after implantation, a monopolar survey was conducted to identify untoward, as well as positive, effects of acute stimulation at any given contact, to be used primarily in determining which electrode configurations to use for chronic stimulation. The surveys, and subsequent DBS adjustments, were made by a single investigator at each site (BDG at BH; DAM at CC). Each of the four contacts on each side was activated singly and in turn, set negative with the INS case set positive. DBS was at $130 \mathrm{~Hz}$, at pulse widths of 90 and $210 \mu \mathrm{s}$. Current amplitudes (calculated on the basis of measured impedance and set voltage) ranged from 2 to $8 \mathrm{~mA}$, depending on electrode impedance. Stimulation at each configuration and setting was tested for approximately $2 \mathrm{~min}$, interspersed with no stimulation periods of equal length. The programmer display was kept of sight of the patient, who remained masked to DBS status. Patients described any adverse effects during the survey, and used 10-point visual analog scales to record any changes in mood, anxiety, or OCD symptoms after each test. A masked rater recorded observations using the same scales.

\section{Chronic DBS}

As with DBS for movement disorders, stimulation adjustment was an iterative process, based on the physician programmer's judgement of therapeutic improvement and tolerability at each rating point. Parameters for chronic DBS were guided most by chronic DBS electrode selection in the Belgian series (Nuttin et al, 2003b) and consultations within 
our collaborative group. That experience, and the results of our intra- and postoperative testing converged to indicate that the best combination of therapeutic benefit and tolerability was generally achieved when the ventral contacts ( 0 and/or 1$)$ were active and negative, although in three patients, contact 2, more dorsal in the capsule, was active as well. Across patients, DBS frequency was 100$130 \mathrm{~Hz}$, pulse width was 90-210 $\mu$ s, and amplitude 8-17 mA. Charge density always remained below the FDA limit of $30 \mu \mathrm{C} / \mathrm{cm}^{2}$. The following electrode contacts were active and negative: 0 ( $8 / 10$ patients), 1 (7/10 patients), and 2 (3/10 patients). Electrode configurations were bipolar (using contact 3 as the anode) in six patients, and monopolar in four. Stimulation was continuous. It was bilateral in $8 / 10$ patients, and unilateral in 2/10 (left and right unilateral in one patient each).

Patients were closely monitored for deterioration in psychiatric status or stimulation-related adverse effects throughout. DBS continued until it was interrupted by stimulator battery depletion, which occurred over a range of 5.5-13 months after the start of chronic DBS across patients. After INS depletion, the devices were replaced in outpatient surgery under local anesthesia.

\section{Concomitant Therapies}

As DBS was used as an adjunctive treatment in a severely affected group, concomitant pharmacotherapy was allowed. Medications were held constant for at least 3 months: SRI (7/10 patients); benzodiazepine (5/10); typical neuroleptic (2/10); atypical neuroleptic (4/10); and anticonvulsant (2/ 10). Patients were also allowed to continue behavior therapy if established at least months before implantation. Medication changes and new or resumed behavior therapy were allowed beginning 6 months after stimulation began. In practice, patients with at least a $25 \%$ improvement in YBOCS OCD severity at 36 months received a mean of $3.4 \pm 0.9$ psychotropic medications at presurgical baseline, and $2.9 \pm 0.6$ at 36 months; in contrast, patients who failed to improve were on a mean of $3.0 \pm 0$ psychotropics at baseline, and $6.0 \pm 1.0$ medications at 36 months.

\section{Outcome Measures}

Data were collected at presurgical baseline, after about 3 weeks of postoperative recovery, but before DBS began, and then at $1,3,6,16,18,24,30$, and 36 months of chronic DBS. Ratings were made by one study nurse at each study site, who was not aware of DBS parameters. The primary outcome measure was the YBOCS. Scores were analyzed as a continuous outcome with repeated-measures analysis of variance (ANOVA, two-tailed). YBOCS OCD severity was also assessed categorically at each rating point.

As clinical experience indicates that depression and anxiety symptoms are highly comorbid with otherwise intractable OCD patients who present for surgery, we used the Hamilton Depression Rating Scale (HDRS)-24 and the Hamilton Anxiety Rating Scale (HARS; Hamilton, 1959) as secondary instruments, analyzed with repeated-measures ANOVA. Global functional status was assessed with the Global Assessment of Functioning (GAF; Hall, 1995) as a continuous measure using repeated-measures ANOVA.
Functional outcomes were also assessed descriptively in four categories: occupation, activities of daily living (ADLs), independent living, and social engagement. Cognitive functioning was assessed before implantation and after chronic DBS with a neuropsychological battery including measures of IQ, visuomotor speed and mental flexibility, verbal and visual learning, memory, and conceptual reasoning. A brief summary of these results is presented below; they are discussed in detail in a forthcoming manuscript (Kubu et al, in preparation).

\section{RESULTS}

\section{OCD Severity after Chronic DBS}

Figure 2 shows that the mean preimplantation baseline YBOCS score $( \pm$ SEM $)$ was $34.6 \pm 0.6$, indicating severe illness. At 3 weeks postoperatively, just before stimulation began, scores were $33.3 \pm 1.0$, indicating no effect of lead insertion alone on OCD severity several weeks after implantation. YBOCS scores decreased during DBS, reaching $22.3 \pm 2.1$ at 36 months (repeated measures overall ANOVA for time: $F(9,63)=7.47 ; p<0.001)$. Most of the improvement occurred over the first months of stimulation; mean YBOCS was $25 \pm 1.6$ at 3 months.

We used a categorical approach to examine changes in YBOCS at the individual level. Table 2 shows the number of study patients in each of three, mutually exclusive, categories from 1 to 36 months after the start of chronic DBS. Those with less than a $25 \%$ reduction in YBOCS compared to presurgical baseline are at left, those with between a 25 and $35 \%$ YBOCS reduction in the center, and those with a $35 \%$ or greater YBOCS reduction are at right. The total $N$ in the right-most column reflects that one patient of the original 10 died (of recurrent cancer) at 9 months, and one patient has just reached the 24-month rating as of this report. The number of responders using the $35 \%$ YBOCS criterion increased from one of 10 at 1 month to four of eight at 36 months. The number of patients with less than a $25 \%$ decrease in YBOCS declined from seven of 10 at 1 month to two of eight at 36 months.

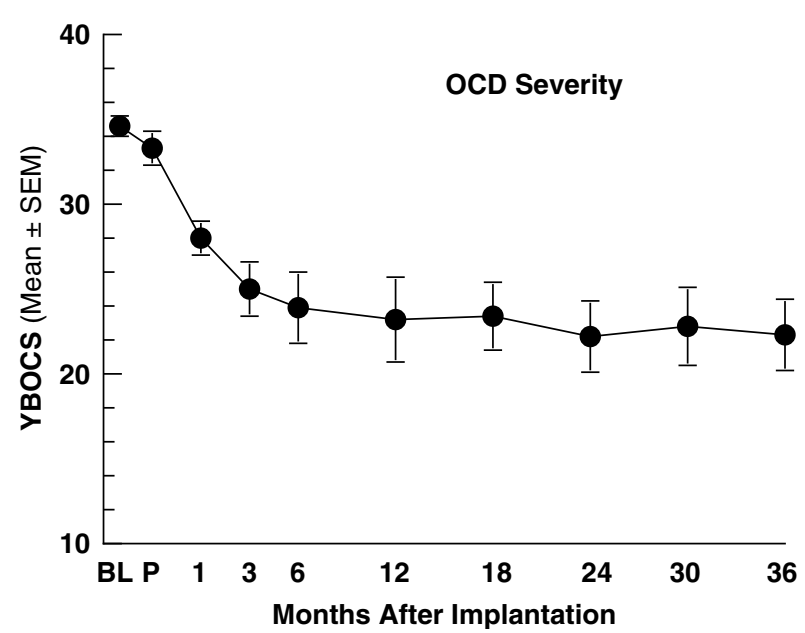

Figure 2 OCD severity (YBOCS) during long-term DBS. 


\section{Comorbid Depressive and Anxiety Symptoms}

Figure 3 shows changes in ratings on the 24-item HDRS-24 (top) and HARS (bottom) during DBS. At presurgical baseline, HDRS-24 mean ( \pm SEM) was $21.1 \pm 1.5$. Three weeks after implantation, but before stimulation, scores were $19.9 \pm 2.0$. Depression scores decreased to $14.7 \pm 2.1$ by 3 months, after which they remained essentially stable. At 36 months, HDRS-24 scores were $15.4 \pm 2.6$ (repeated measures ANOVA for time: $\mathrm{F}(9,63)=2.53 ; p=0.015)$.

Table 2 Categorical Responses during Long-Term DBS for OCD

\begin{tabular}{|c|c|c|c|c|}
\hline $\begin{array}{l}\text { DBS } \\
\text { duration }\end{array}$ & $\begin{array}{c}<25 \% \\
\text { YBOCS } \downarrow \\
\text { (no. of pts, \%) }\end{array}$ & $\begin{array}{c}\geqslant 25<35 \% \\
\text { YBOCS } \downarrow \\
\text { (no. of pts, \%) }\end{array}$ & $\begin{array}{c}\geqslant 35 \% \\
\text { YBOCS } \downarrow \\
\text { (no. of pts, \%) }\end{array}$ & $\begin{array}{c}\text { Total } \\
\mathbf{N}\end{array}$ \\
\hline I month & $7(70)$ & $2(20)$ & I (10) & 10 \\
\hline 3 months & $5(50)$ & $2(20)$ & $3(30)$ & 10 \\
\hline 6 months & $4(40)$ & $4(40)$ & $2(20)$ & 10 \\
\hline 12 months & $4(44)$ & $2(22)$ & $3(33)$ & 9 \\
\hline 18 months & $3(33)$ & $3(33)$ & $3(33)$ & 9 \\
\hline 24 months & $2(22)$ & $3(33)$ & $4(44)$ & 9 \\
\hline 30 months & $3(38)$ & I (I2) & $4(50)$ & 8 \\
\hline 36 months & $2(25)$ & $2(25)$ & $4(50)$ & 8 \\
\hline
\end{tabular}
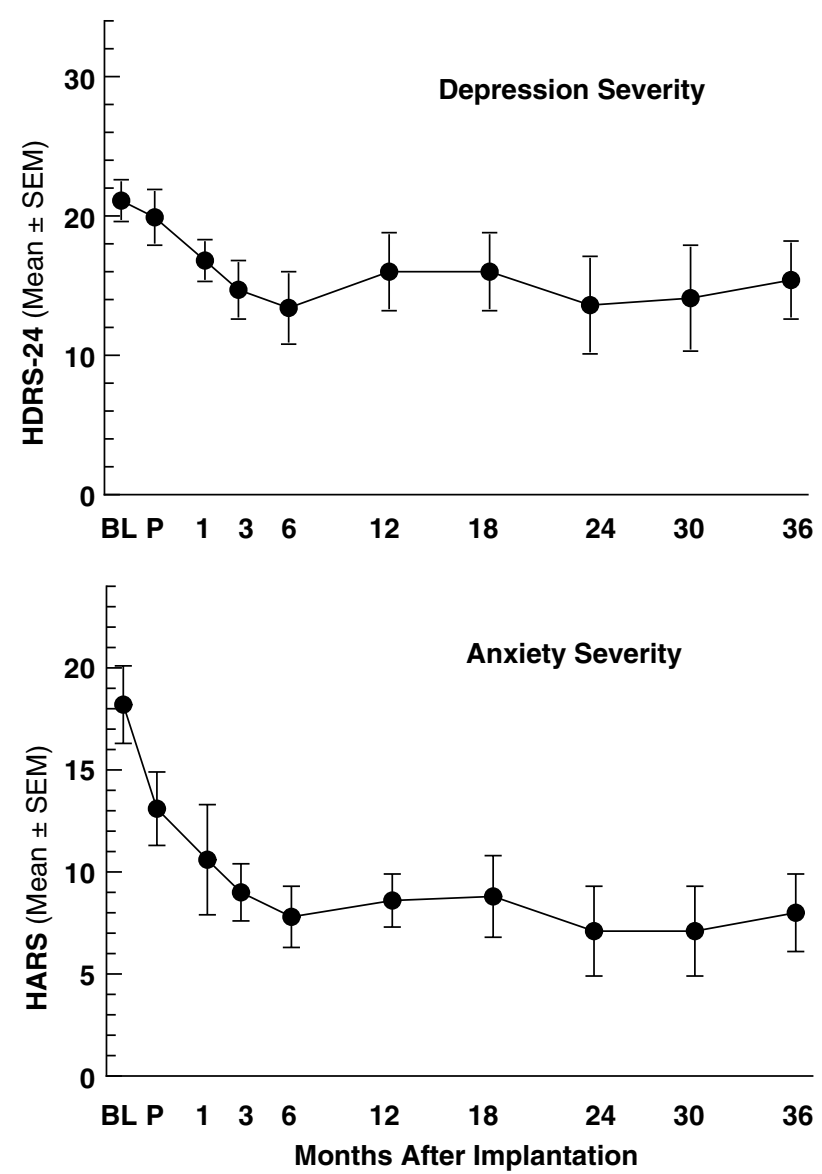

Figure 3 Depression (top) and anxiety (bottom) severity ratings during long-term DBS for OCD.
Anxiety measured by the HARS also improved during longterm DBS. HARS scores were $18.2 \pm 1.9$ at presurgical baseline, decreasing to $13.1 \pm 1.83$ weeks after implantation (before DBS began). After 3 months of DBS, mean HARS ratings were $9.0 \pm 1.4$. After that they changed little. At 36 months, HARS scores were $8.0 \pm 1.9$ (repeated measures ANOVA for time: $\mathrm{F}(9,63)=5.70 ; p<0.001)$.

\section{Global Functioning}

Figure 4 shows that mean scores on the GAF improved significantly over time during long-term DBS, from $36.6 \pm 1.5$ at presurgical baseline to $53.8 \pm 2.5$ at 36 months (repeated measures ANOVA: $\mathrm{F}(9,63)=7.57 ; p<0.001)$. Table 3 includes a description of functioning before and after stimulation in four categories: work or school, ADLs, ability to live independently, and social engagement. No patient was working or in school before DBS; six were during chronic stimulation. Ability to perform ADLs independently was markedly impaired in seven patients at baseline; this was true of one patient during DBS. No patient lived independently before DBS, whereas six did so afterwards. With the exception of the patient who died owing to recurrent breast cancer 9 months after implantation, social engagement improved in eight of nine patients, to varying degrees ranging from greater social contact to becoming engaged to marry.

\section{Adverse Effects}

Potential complications of DBS can be separated into those related to surgical implantation, device failure, and the stimulation itself. There were no device failures beyond the expected stimulation interruptions owing to INS battery depletion or owing to device shutoff if the magnetic switch on the Soletra INS device was tripped by a metal or theft detector.

Adverse effects of implantation. One patient had a small asymptomatic intracerebral hemorrhage after lead insertion. This appeared to result from blood from a ruptured superficial bridging vein tracking down the insertion guide cannula. The hemorrhage resolved on repeat CT scans within days after implantation; no clinical intervention was

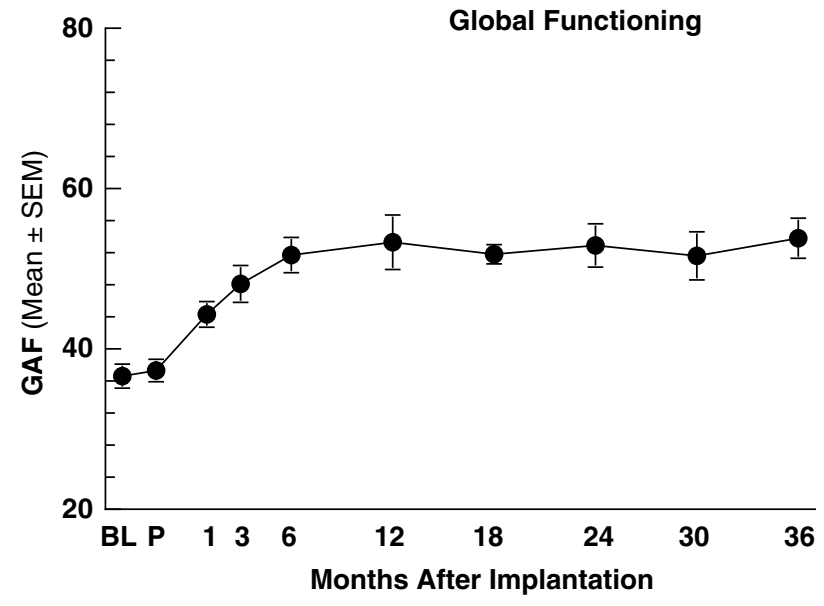

Figure 4 Global functioning during long-term DBS. 
Table 3 Clinical Assessment of Functioning before and during Chronic DBS

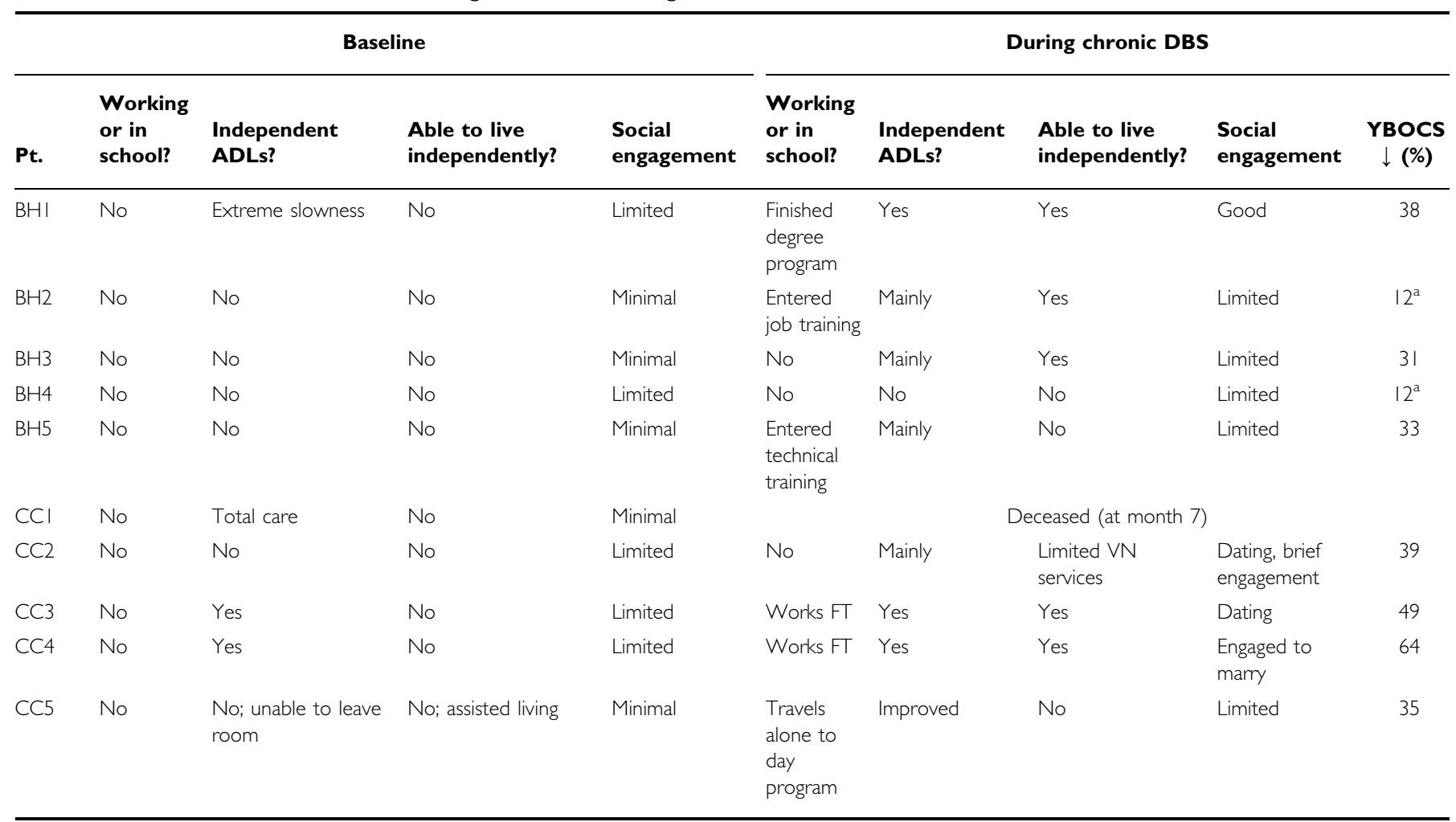

The two patients who discontinued stimulation before the 36-month end point.

required. Another patient had a single intraoperative generalized tonic-clonic seizure after lead implantation. She was treated with prophylactic phenytoin for 30 days afterwards. Seizures have not recurred during the following 3 years. One patient, who had comorbid insulin-dependent diabetes mellitus, developed a superficial surgical wound infection after implantation, successfully treated with antibiotics.

Adverse effects of stimulation. Acute adverse effects of DBS included transient sadness, anxiety, and euphoria or giddiness. Patients also experienced motor effects. One displayed a unilateral 'smile' contralateral to the side of test stimulation (see Nuttin et al (2003b) and Okun et al (2004) for descriptions of similar events in different series). One patient developed jaw muscle tightness associated with dysarthria. Another patient developed a transient, epigastric, 'physical sensation of sadness' lasting about $30 \mathrm{~s}$, unaccompanied by a sad mood, which abated without parameter change. Another patient had stimulation-related olfactory and gustatory sensations, described as a 'chemical' or 'metallic' smell alone, or a smell plus taste (at higher amplitudes). These effects occurred within seconds to minutes of DBS onset. They all reversed, typically within seconds and always within minutes, usually when DBS was stopped or parameters were changed, but sometimes spontaneously.

Mood elevation/hypomania. Five patients developed a transient elevated mood associated with noticeably increased energy, speech production, and spontaneity of social interactions, but without an increase in behavioral impulsivity. In four of these cases, the events occurred within minutes after DBS began, lasted for hours and abated without parameter change, and so did not meet DSM-IV criteria for a hypomanic episode. In the fifth case, this behavioral state, which had equally rapid onset, lasted for 4 days and reversed within minutes after DBS parameter change.

A few stimulation-related adverse events developed or persisted over days. One patient, after an increase in amplitude at the distal contact (0), developed brief memory experiences for events surrounding the surgery itself. These recurred several times a day over several days and ceased when she reported them and parameters were changed. A patient with insulin-dependent diabetes mellitus had an unexplained syncopal episode while on stimulation, which was possibly owing to hypoglycemia.

The adverse effects above, particularly increased anxiety and/or flushing (which was seen in five of 10 patients), were most common when the most distal electrode contact 0 was active and negative. Two of these patients experienced increased anxiety during stimulation of contact 1 as well. Anxiety was more frequent with monopolar than with bipolar stimulation. The lowest monopolar stimulation amplitude producing increased anxiety was $3 \mathrm{~mA}$ in one patient. In the four others, monopolar stimulation of contact 0 resulted in increased anxiety at DBS intensities of 5-7 mA. In contrast, bipolar stimulation produced flushing only when the amplitude reached $13 \mathrm{~mA}$, and only in one patient. Similar anxiogenic effects of monopolar stimulation of the distal contact at this DBS site have been reported in one OCD case (Shapira et al, 2006). 
None of these adverse effects persisted. Although most adverse effects (like most positive effects) were induced by stimulation of the ventral two contacts, one patient developed verbal perseveration lasting $30 \mathrm{~s}$ when the dorsal contacts were stimulated during intraoperative testing. Amplitudes which produced these adverse effects and those associated with therapeutic benefit were generally similar. However, if setting a particular contact negative repeatedly produced adverse effects on acute testing, this was avoided during chronic stimulation. There were no sensorimotor changes noted on detailed postoperative neurological examinations.

Effects of DBS interruption. Six of the nine patients who experienced DBS interruption (mainly owing to stimulator battery depletion) became acutely aware of a more depressed mood. Patients always informed the investigators when this occurred, even though they were unaware of stimulator status at these times. The clinical worsening typically abated somewhat over several days, and reversed after DBS was restarted. Five of these patients also had an acute worsening in OCD symptoms after DBS interruption, although this was clinically less rapid, and less marked than the change in their affective state. We obtained HDRS scores within a week of DBS interruption for the five Butler patients. The three patients who had long-term improvement in OCD all had worsened HDRS depression ratings after stimulation interruption. The mean of three consecutive HDRS ratings before DBS interruption in these three patients was $12 \pm 4.7$ (SEM), and increased to $22.7 \pm 1.5$ after DBS stopped. In contrast, HDRS scores for the two Butler patients whose OCD did not improve after chronic DBS were unchanged after stimulation interruption: $20.9 \pm 2.2$ (mean of the three consecutive ratings before interruption) $v s 19 \pm 0$ in the week after DBS stopped.

Suicidality. Patients were monitored closely for suicidality throughout. No patient became acutely suicidal when DBS was interrupted. Moreover, for no patient did ratings on the suicidality item of the HRDS-24 exceed a score of 2 ('wishes he were dead or any thoughts of possible death to self) during chronic DBS.

Cognition. All patients completed neuropsychological assessments before implantation and after a mean of approximately 10 months of chronic DBS. Analysis at the individual patient level using practice corrected reliable change indices found no pattern of pervasive decline or improvement in any one patient. The group analyses revealed statistically significant improvements in recall of prose passages during chronic stimulation. There were no significant performance declines at the group level. Overall, the results suggest that the cognitive adverse event burden of DBS for OCD at this target and parameters is relatively benign (Kubu et al, in preparation), similar to effects observed in the Belgian series (Gabriels et al, 2003).

\section{DISCUSSION}

This study, in agreement with Nuttin et al's (2003) study of long-term effects in four OCD patients, suggests that DBS at the $\mathrm{VC} / \mathrm{VS}$ target is associated with long-term improvement in symptomatology and functioning in OCD patients with chronic severe illness, who had failed to respond to behavior therapy and medication. We found that OCD improved from the severe illness at baseline (required by our entry criteria) to a mean score reflecting moderate illness during DBS extending over 3 years. Six of the eight patients who reached the 3-year rating point experienced a $25 \%$ or greater reduction in YBOCS severity compared to baseline (the response criterion most common in pharmacological trials). Four of the eight patients met or exceeded a $35 \%$ YBOCS reduction, regarded as a stringent response criterion in medication treatment studies. Moreover, in such studies, patients are not selected, as here, for marked treatment resistance. As expected based on prior DBS work, depressive and anxiety symptoms, which are in our experience prevalent in this group, improved along with core OCD symptoms. However, clinically significant OCD, depressive, and anxiety symptoms persisted in most patients. Nevertheless, most had both symptomatic improvement and gains in real-world functioning. Baseline YBOCS severity, which was clustered narrowly in the severe range (mean \pm SEM: $34.6 \pm 0.6$ ), did not predict outcome in this sample.

Although DBS was generally well tolerated, there were a number of adverse events. Those owing to surgical implantation included an asymptomatic intracerebral hemorrhage, a single intraoperative seizure, and an infected surgical incision. Although surgical complications of device implantation are potentially serious, in our experience the psychiatric adverse events of DBS, mainly DBS-induced acute mood elevation, which in one case met the DSM-IV criteria for a hypomanic episode, and depressive and OCD symptom worsening if DBS is interrupted, may impose the greatest risks.

Although the prominent affective impacts of VC/VS DBS represent a potential risk of this procedure, affective change might also be involved in the long-term improvement in OCD. All seven patients whose OCD improved at $24-36$ months had either rapid mood elevation when DBS began (five of seven) or affective worsening when stimulation was interrupted (six of seven). Most had both. Clinically, we observed notably enhanced motivation to engage in goaldirected activities during DBS. This included behavior therapy for OCD, which all had attempted unsuccessfully before the procedure. This may have been a key factor in their clinical progress. The importance of change in comorbid depression in OCD outcomes can be better determined in a larger study.

In this study, no patient attempted suicide or became acutely suicidal during DBS. This was also true when stimulation was interrupted by INS battery depletion, even though depression worsening was the first symptomatic change we observed after DBS stopped. Elsewhere, patients have died by suicide during stimulation for movement disorders (Burkhard et al, 2004), or, in one case, DBS for OCD at a target similar but not identical to ours (Abelson et al, 2005). As a group, patients referred for OCD neurosurgery suffer profound demoralization after years of severe impairment that is unrelieved by treatment. Most have comorbid depressive syndromes as well. Several patients in our study had suicidal thinking before enrollment. At our center and others, suicides have occurred in 
patients during the evaluation process, or after their acceptance as candidates but before surgery (L Gabriels, personal communication 1/06; S Rasmussen, unpublished data). Patients should be considered as having potentially high suicide risk at all phases of evaluation and treatment. It is abundantly clear that the psychiatric use of DBS requires dedicated interdisciplinary teams expert in patient selection, implantation, stimulation, and long-term patient monitoring. Psychiatric leadership of such teams is essential.

\section{Comment on Target Selection}

The overall rationale for DBS at the VC/VS target is to modulate the corticobasal circuitry that is consistently implicated in OCD symptoms and in the response to treatment (Rauch, 2003), although the mechanisms by which DBS accomplishes neuromodulation remain unknown and are a subject of very active research (Benabid et al, 2002; McIntyre and Grill, 2002; Montgomery and Baker, 2000; Vitek, 2002). The VC/VS stimulation site is more posterior than that for the capsulotomy lesions on which it was originally based. The VC/VS site is also more posterior than the target used in other DBS studies where the anterior capsule and/or the adjacent ventral striatum were stimulated (Abelson et al, 2005; Aouizerate et al, 2005). Our target is similar in anterior-posterior location to the nucleus accumbens stimulation site used by Sturm et al (2003), although they used a different surgical trajectory and electrode design. In addition, it is likely that there is an optimum dorsoventral location where VC/VS electrode placement gives the best therapeutic effects. In this regard, an intriguing albeit preliminary finding from ongoing detailed localization of the midpoints of ventral contacts 0 and 1 in this study, correlating contact locations with clinical response, suggests that activating contacts closest to the junction of the ventral anterior capsule fibers and the anterior commissure might give the best effects (A Machado et al, unpublished data).

\section{Limitations}

This study was not controlled, so a placebo effect is possible. The persistence of benefit for 3 years argues against a placebo effect, as does the symptomatic worsening when DBS stopped when stimulator batteries became depleted. Although held constant before implantation and for the first 3 months of DBS, medication regimens differed across patients, another potential source of variability. It was not feasible to maintain constant medication regimens over a 3-year period. The number of medications was reduced or remained stable over time in patients who improved during the study, whereas the number of medications received increased in the nonresponders. Medication changes thus seem unlikely to account for the therapeutic effects observed. An effect of lead insertion itself (a 'microlesion') is also possible, and a sham-controlled study is necessary to more definitively address this question. We think, however, that an insertion effect is very unlikely, as OCD symptoms and functional status were essentially unchanged after implantation but before DBS started. Moreover, symptomatic improvements during VC/
VS DBS were not maintained during masked stimulation withdrawal in one study (Gabriels et al, 2003). Furthermore, stimulation interruption owing to battery depletion, which was not known in advance by patients or investigators, and not detectable by patients (thus essentially masked), was regularly associated with symptom worsening in both the Belgian series (Nuttin et al, 2003b) and here. Although both the GAF and clinical description indicated that most patients made meaningful gains, more detailed assessments of functioning and quality of life are needed. However, the fact that in five of nine patients moved to independent living status is highly encouraging. To assess such realworld functioning as well as symptomatology, future studies should also include a non-surgical comparator group matched for OCD severity and refractoriness.

The sample, although larger than the others published to date, was nonetheless small. A larger DBS study using a controlled design would permit an investigation of possible clinical predictors of response. Here, baseline YBOCS severity, which was clustered tightly in the severe range, did not predict response. It is important to spare patients the surgical risks of implantation and the primarily psychiatric risks of stimulation if it can be determined that there is a subgroup that is substantially less likely to respond to this intervention. For example, for OCD, there is increasing evidence that symptom subtypes (eg, Leckman et al, 1997) may be mediated by different neural circuitry (Mataix-Cols et al, 2004; Saxena et al, 2004). This raises the question of whether DBS targeting thalamocortical circuitry might more effectively treat some OCD symptoms than others. Here, we can only note that the two patients where DBS was discontinued owing to inadequate benefit both primarily had symptoms we have described as 'incompleteness', that is, the need to repeat actions until a sense of completeness or rightness is obtained (Rasmussen and Eisen, 1992). However, four other patients in this series who had the symptom of incompleteness did improve after DBS.

Importantly, a larger patient group is also needed to determine if there is a relationship between precise postimplantation lead location and response that could be used to further improve the therapy. Larger samples are needed to adequately evaluate DBS at the VC/VS target against alternative electrode placements, such as the subthalamic nucleus site, where DBS has improved comorbid OCD symptoms in patients with Parkinson disease (Fontaine et al, 2004; Mallet et al, 2002).

DBS and neuroimaging. The basic rationale for VC/VS DBS in OCD is derived from the empirical results of lesion procedures and from neuroanatomical models of OCD that have emerged from neuroimaging research (Rauch, 2003). An initial study using O15-PET imaging in patients from this series found that acute high-frequency DBS increased perfusion of orbitofrontal cortex, anterior cingulate cortex, striatum, pallidum, and thalamus compared to control conditions. Acute DBS at the VC/VS target thus was associated with activation of circuitry implicated in OCD (Rauch et al, 2006). Another potential use of imaging is response prediction. An intriguing finding from the Belgian series of OCD patients undergoing VC/VS stimulation is that preimplantation metabolism in the subgenual cingulate 
cortex on FDG-PET was highly and positively correlated with the therapeutic outcome of DBS for OCD (Van Laere et al, 2006). Replication of this finding in a larger sample would help to determine if such measures are robust enough predictors to potentially see clinical use in patient selection.

\section{Conclusion}

This long-term study suggests that VC/VS DBS has encouraging therapeutic effects, supporting the findings of earlier short-term and small-scale studies. A more definitive test of the efficacy and tolerability of DBS will require a larger controlled trial. By virtue of its focality and adjustability, DBS also opens a unique window on the neurocircuitry involved in the pathophysiology of OCD, and possibly of related illnesses as well.

\section{ACKNOWLEDGEMENTS}

This work was supported by an Independent Investigator Award from the National Alliance for Research on Schizophrenia and Depression (BDG, SAR); The National Institute of Mental Health (MSO and WKG); and research funding from Medtronic Inc. (BDG, SAR, GMF, DAM, ARR). We thank Bart Nuttin, Volker Sturm, Doris Lenartz, Kelly Foote, Andre Machado, and Robert Gross for consultations on neurosurgical issues; Loes Gabriels, Paul Cosyns, NA Shapira, Anthony Grace, and Erwin Montgomery for consultations on electrical stimulation and clinical issues in this population; Paul Stypulkowski, Mark Rise, and Roy Testerman for technical advice on the DBS devices; William Wong, Ronald Gaertner, and Richard Marsland for invaluable assistance in patient care; Suzanne Haber, Scott Rauch, and Darin Dougherty for discussions of neurocircuitry and neurosurgery in this population; and Rouba Youssef, Katherine Rowinski, Erin Einbinder, and Natalie Sykuta for help with data management and analysis.

\section{REFERENCES}

Abelson JL, Curtis GC, Sagher O, Albucher RC, Harrigan M, Taylor SF et al (2005). Deep brain stimulation for refractory obsessivecompulsive disorder. Biol Psychiatry 57: 510-516.

Anderson D, Ahmed A (2003). Treatment of patients with intractable obsessive-compulsive disorder with anterior capsular stimulation. Case report. J Neurosurg 98: 1104-1108.

Aouizerate B, Cuny E, Martin-Guehl C, Guehl D, Amieva H, Benazzouz A et al (2004). Deep brain stimulation of the ventral caudate nucleus in the treatment of obsessive-compulsive disorder and major depression. Case report. J Neurosurg 101: 682-686.

Aouizerate B, Martin-Guehl C, Cuny E, Guehl D, Amieva H, Benazzouz A et al (2005). Deep brain stimulation for OCD and major depression. Am J Psychiatry 162: 2192.

Benabid AL, Benazzous A, Pollak P (2002). Mechanisms of deep brain stimulation. Mov Disord 17: S73-S74.

Burkhard PR, Vingerhoets FJ, Berney A, Bogousslavsky J, Villemure JG, Ghika J (2004). Suicide after successful deep brain stimulation for movement disorders. Neurology 63: 2170-2172.

Dougherty DD, Baer L, Cosgrove GR, Cassem EH, Price BH, Nierenberg AA et al (2002). Prospective long-term follow-up of 44 patients who received cingulotomy for treatment-refractory obsessive-compulsive disorder. Am J Psychiatry 159: 269-275.

First MB, Spitzer RL, Gibbon M, Williams JBW (2001). The Structured Clinical Interview for DSM-IV. Biometrics: New York.

Fontaine D, Mattei V, Borg M, von Langsdorff D, Magnie MN, Chanalet $S$ et al (2004). Effect of subthalamic nucleus stimulation on obsessive-compulsive disorder in a patient with Parkinson disease. Case report. J Neurosurg 100: 1084-1086.

Gabriels L, Cosyns P, Nuttin B, Demeulemeester H, Gybels J (2003). Deep brain stimulation for treatment-refractory obsessive-compulsive disorder: psychopathological and neuropsychological outcome in three cases. Acta Psychiatr Scand 107: 275-282.

Goodman WK, Price LH, Rasmussen SA, Mazure C, Delgado P, Heninger GR et al (1989). The Yale-Brown obsessive compulsive scale. II. Validity. Arch Gen Psychiatry 46: 1012-1016.

Greenberg BD, Price LH, Rauch SL, Friehs G, Noren G, Malone D et al (2003). Neurosurgery for intractable obsessive-compulsive disorder and depression: critical issues. Neurosurg Clin N Am 14: 199-212.

Hall RC (1995). Global assessment of functioning. A modified scale. Psychosomatics 36: 267-275.

Hamilton M (1959). The assessment of anxiety states by rating. $\mathrm{Br} J$ Med Psychol 32: 50-55.

Hamilton M (1967). Development of a rating scale for primary depressive illness. Br J Soc ClinPsychol 6: 278-296.

Leckman JF, Grice DE, Boardman J, Zhang H, Vitale A, Bondi C et al (1997). Symptoms of obsessive-compulsive disorder. Am J Psychiatry 154: 911-917.

Mallet L, Mesnage V, Houeto JL, Pelissolo A, Yelnik J, Behar C et al (2002). Compulsions, Parkinson's disease, and stimulation. Lancet 360: 1302-1304.

Mataix-Cols D, Wooderson S, Lawrence N, Brammer MJ, Speckens A, Phillips ML (2004). Distinct neural correlates of washing, checking, and hoarding symptom dimensions in obsessive-compulsive disorder. Arch Gen Psychiatry 61: 564-576.

McIntyre CC, Grill WM (2002). Extracellular stimulation of central neurons: influence of stimulus waveform and frequency on neuronal output. J Neurophysiol 88: 1592-1604.

Montgomery Jr EB, Baker KB (2000). Mechanisms of deep brain stimulation and future technical developments. Neurol Res 22: 259-266.

Nuttin B, Cosyns P, Demeulemeester H, Gybels J, Meyerson B (1999). Electrical stimulation in anterior limbs of internal capsules in patients with obsessive-compulsive disorder. Lancet 354: 1526.

Nuttin B, Gybels J, Cosyns P, Gabriels L, Meyerson B, Andreewitch $S$ et al (2003a). Deep brain stimulation for psychiatric disorders. Neurosurg Clin N Am 14: xv-xvi.

Nuttin BJ, Gabriels LA, Cosyns PR, Meyerson BA, Andreewitch S, Sunaert SG et al (2003b). Long-term electrical capsular stimulation in patients with obsessive-compulsive disorder. Neurosurgery 52: 1263-1272; discussion 1272-1274.

Okun MS, Bowers D, Springer U, Shapira NA, Malone D, Rezai AR et al (2004). What's in a 'smile?' Intra-operative observations of contralateral smiles induced by deep brain stimulation. Neurocase 10: 271-279.

Rasmussen SA, Eisen JL (1992). The epidemiology and clinical features of obsessive compulsive disorder. Psychiatr Clin N Am 15: 743-758.

Rauch SL (2003). Neuroimaging and neurocircuitry models pertaining to the neurosurgical treatment of psychiatric disorders. Neurosurg Clin N Am 14: 213-223, vii-viii.

Rauch SL, Dougherty DD, Malone D, Rezai A, Friehs G, Fischman AJ et al (2006). A functional neuroimaging investigation of deep brain stimulation in patients with obsessive compulsive disorder. J Neurosurg 104: 558-565. 
Saxena S, Brody AL, Maidment KM, Smith EC, Zohrabi N, Katz E et al (2004). Cerebral glucose metabolism in obsessive-compulsive hoarding. Am J Psychiatry 161: 1038-1048.

Shapira NA, Okun MS, Wint D, Foote KD, Byars JA, Bowers D et al (2006). Panic and fear induced by deep brain stimulation. J Neurol Neurosurg Psychiatry 77: 410-412.

Sturm V, Lenartz D, Koulousakis A, Treuer H, Herholz K, Klein JC et al (2003). The nucleus accumbens: a target for deep brain stimulation in obsessive-compulsive- and anxiety-disorders. $J$ Chem Neuroanat 26: 293-299.

Van Laere K, Nuttin B, Gabriels L, Dupont P, Rasmussen S, Greenberg BD et al (2006). Metabolic imaging of anterior capsular stimulation in refractory obsessive compulsive disorder: a key role for the subgenual anterior cingulate and ventral striatum. J Nuc Med 47: 740-747.

Vitek JL (2002). Mechanisms of deep brain stimulation: excitation or inhibition. Mov Disord 17: S69-S72. 